\title{
Big Data Compression Technology Based on Internet of Vehicles
}

\author{
https://doi.org/10.3991/ijoe.v15i01.9773 \\ Guohua Xiong \\ Guandong Construction Polytechnic, Guangdong, China \\ guohuaxiongzud291@163.com
}

\begin{abstract}
To ensure the high efficiency of the development of car networking technology, large data compression technology based on car networking was studied. First, RFID technology and vehicle networking, big data technology in vehicle networking, RFID path data compression technology in the Internet of vehicles were introduced. Then, RFID path data compression verification experiments were performed. The results showed that when the data volume was relatively small, there was no obvious change in the compression ratio under the fixed threshold and the threshold change. However, when the amount of data gradually increased, the compression ratio under the condition of changing the threshold was slightly higher than the fixed threshold. Therefore, RFID path big data processing is feasible, and compression technology is efficient.
\end{abstract}

Keywords - Car networking, big data compression technology, RFID technology

\section{$1 \quad$ Introduction}

RFID (Radio Frequency Identification) is a non-contact automatic identification technology realized by radio frequency communication, which can quickly and efficiently read and store object information during motion. Car networking means that the electronic tag loaded on the vehicle is recognized by RFID. On the information network platform, attribute information of all vehicles including static and dynamic information is extracted and utilized. According to different users, the needs of the functions are different. The vehicle's operating conditions and surrounding environment are effectively regulated and integrated services are provided.

As a new term generated in the context of the development of the Internet of Things, car networking is the specific application of the Internet of Things in the field of transportation. The definition of the Internet of Things refers to the interconnection of the Internet with information sensing devices such as RFID. Information is exchanged. Intelligent identification, tracking, monitoring, location, and management of items are implemented. Car networking contributes to the convergence of technologies and applications in modern fields such as ICT, automotive electronics, 
intelligent transportation and in-vehicle information services. Using modern information communication integrated technology applications such as vehicle electronic identification, sensors, wireless mobile communications, satellite positioning and mass data processing, all road traffic infrastructure in the network as well as basic attributes, static and dynamic information of the vehicle are collected and identified. Through the network information system, information is intelligently managed and served. It is widely used in energy conservation and emission reduction, logistics and transportation, traffic safety, traffic congestion, transportation services, emergency rescue, intelligent toll, urban management and personal information integrated services.

\section{State of the Art}

To facilitate the management of RFID data, Kim et al. [1] proposed two important concepts, namely flow cubes and flow graphs. An efficient flow cube model was built. In the model, data cube technology is used to solve the problem of granularity and multi-dimensional data mining in RFID path data. A workflow method that considers the probability is proposed. In the RFID data mining part, the closed path condition probability is mined by the frequent closed item set method. Grishentsev et al. [2] pointed out that the frequent pattern mining method for RFID path data mainly mines frequent patterns based on the intermediate data sets generated by the original database. In the process of mining frequent patterns, different RFID electronic tag characteristics are described by using fuzzy rules. Heo and Jeon [3] proposed a method for RFID data storage and query in the supply chain. The RFID path data is encoded using a prime coding method. After encoding, the RFID path data effectively reduces the amount of RFID data storage, which facilitates querying the RFID path. This method is applied in the supply chain. The storage and query efficiency of RFID path data is greatly improved. He et al. [4] mainly studied the frequent pattern mining of RFID path data. The RFID path data for activity monitoring is managed. Amadeo et al. [5] conducted in-depth research on RFID path data mining. A path coding and path mining method for identifying path information by path coding pid is proposed. The storage space is effectively reduced, which makes the retrieval of the path more convenient. Chen et al. [6] proposed an RFID data cleaning method that sets the noise threshold. Combined with the characteristics of RFID trajectory data, a trajectory data filling algorithm based on motion aging is proposed. Checko et al. [7] first preprocessed the path data in the RFID path clustering process. It includes path cuts and path sequence comparisons. After preprocessing, a density-based path-clustering algorithm (DBPC) and a path hierarchical clustering algorithm (PHC) are proposed. In the frequent pattern mining of RFID paths, the path CloSpan and CFPM, algorithms are proposed. Da et al. [8] proposed a frequent path based on the time model, which is more conducive to the cleaning of composite events. At the same time, the sliding window of the time decay mechanism is applied to the mining method of frequent path events, the path-mining tree PM-Tree is established, and the latest path information in the RFID data is maintained by PM-Tree. 
In summary, the above research is mainly aimed at the cleaning of RFID data, storage compression of RFID data, detection of RFID events, clustering analysis of RFID data and frequent pattern mining. However, there is no related research on the integration of car networking technology. Therefore, according to the above research status, the big data compression technology based on car networking is studied. Combined with the characteristics of the RFID path data in car networking, the collected massive path data is compressed and experimentally simulated.

\section{$3 \quad$ Methodology}

\subsection{RFID technology and car networking}

Using GPS and Beidou and other positioning technologies and wireless transmission technologies such as GPRS and $3 \mathrm{G}$, the reader reads the RFID electronic tag information loaded on the vehicle. The running state and driving information of the vehicle are extracted, and parameters useful to the user, such as the driving position of the vehicle, are acquired, and information transmission and sharing are realized. Using RFID technology and sensing technology, the status of transportation infrastructure such as roads and bridges is acquired. Under the support of the Internet information platform, the vehicle's operating conditions are monitored and various traffic integrated information services are provided.

RFID technology is an automatic identification technology based on wireless communication. The reader in the RFID system uses the radio signal to communicate with the electronic tag, acquires the tag information and provides it to the subsequent processing system, and completes the task of automatically identifying and controlling the reading and writing of the data.

As the data carrier of the radio frequency identification system, the electronic tag is mainly composed of a tag antenna and a tag-dedicated chip. According to different electronic tag power supply methods, it can be divided into three types: active electronic tags, passive electronic tags and semi-passive electronic tags. The internal battery is equipped with an active electronic tag, the battery without a built-in battery is a passive electronic tag, and the part that relies on the battery is a semi-passive electronic tag. According to different frequencies, electronic tags can be divided into four categories: low frequency, high frequency, ultra-high frequency and microwave electronic tags.

Car networking is a mobile ad hoc network. Therefore, it has some of the same characteristics as the self-organizing network. For example, the node characteristics have huge storage capacity and computing power, and its mobile characteristics are rapid change of network topology, fast moving speed of nodes, and predictable moving routes. Its data stream characteristics can predict real-time road condition information. The biggest value of car networking is the ability to extract useful vehicle information and effectively process the information to solve the road congestion problem. According to statistics, car networking has the following advantages. First, traffic congestion is reduced by $60 \%$. Second, short-distance 
transportation efficiency has increased by about $70 \%$. The capacity of existing road networks has been increased by 2-3 times. It can not only improve production and living efficiency, reduce operating costs, but also make full use of social public resources, so that the government's management funds are greatly reduced.

Radio frequency identification technology can quickly and accurately identify high-speed moving targets. Compared with traditional traffic information collection technology, radio frequency identification technology has unparalleled advantages, which has become the main means of future traffic information collection and supervision. Its wide application in traffic management has finally become the development trend of intelligent transportation in the future.

\subsection{Big data technology for car networking}

The data platform support system is responsible for storing and processing the vast amounts of data generated by on-grid vehicles and facilities. At the same time, other service-based data is integrated, which provides support for intelligent traffic management and in-vehicle information services. Cloud computing technology will be one of the important supporting technologies for data platform support systems. The Traffic Management Center has an extensive database and data analysis capabilities to store and analyze data transmitted from roadside facility systems. The corresponding control command is sent according to the analysis result.

The platform support mainly provides technical support for the upper service entities in the car networking system. It mainly includes cloud search with intelligent semantic analysis, rapid collection and release of information, storage of massive geographic information, manual processing based on the combination of web crawling technology and Geocoding, and automatic updating of data by computer and establishment of publishing system. Using user behavior data, the data warehouse is built and user behavior motives are analyzed. The platform combines current traffic conditions with historical data, seamlessly links pedestrian navigation and car navigation, and further develops dynamic navigation based on real-time traffic. In the car networking technology white paper, the car networking system is divided into six parts according to the technical system, as shown in Figure 1. The massive data processing in the platform is mainly the preprocessing, storage and data analysis of related data such as vehicles and road conditions.

Data preprocessing: Since information acquisition and transmission may cause errors, the cloud platform system first needs to perform data preprocessing after receiving the number, including data deduplication, filtering, and calibration. Many car networking typical applications have real-time processing features. The car networking platform should support the real-time processing and decision making of massive amounts of data that is continuously input in the form of a stream.

Storage of data: Due to the huge amount of data collected, the cloud platform needs to design a corresponding storage system for the characteristics of the car networking application itself. By applying redundant storage, tiered storage, caching, indexing, compression and other technical means, the data can be read, written, updated, and 
queried, and an overall storage scheme combining hardware and software is established.

Data analysis: The analysis and mining of massive data requires the establishment of a corresponding data mining and analysis platform to support the drilling and statistical analysis of different dimensions of the data. It also provides support for domain-specific analysis needs such as traffic flow forecasting, travel time estimation, mobile trajectory analysis, and geographic correlation analysis. Car networking technology system is shown in Figure 1.

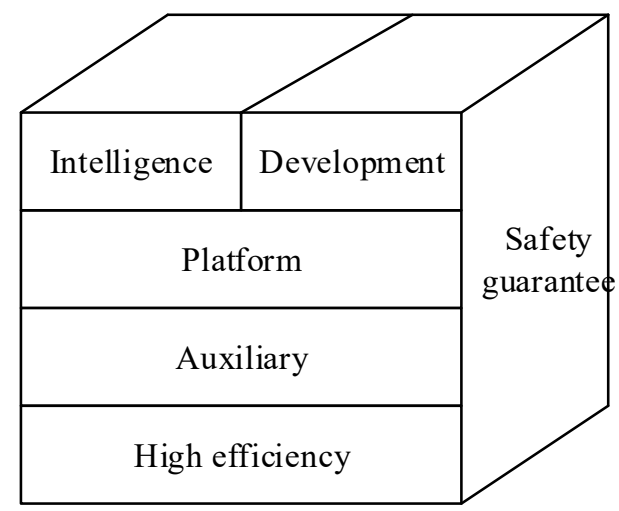

Fig. 1. Car networking technology system

\subsection{The generation and characteristics of RFID path data}

RFID systems generally consist of three main parts: an electronic tag, a reader, and an antenna. When the system works, the reader first sends the command signal to the electronic tag via the antenna. Then, after the tag is responded, the information stored inside the tag is backscattered to the antenna, and the antenna receives the returned information and hands it to the reader for identification operation. Finally, the result of the identification is passed to the computer system, and the recognition process is completed. The composition of the RFID system is shown in Figure 2.

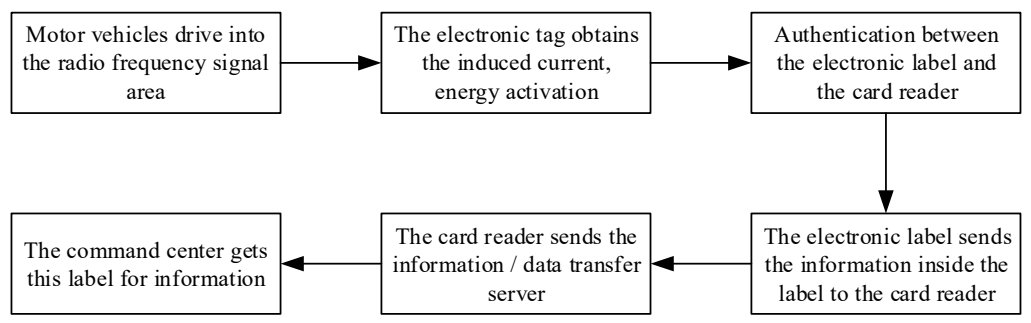

Fig. 2. Schematic diagram of RFID system 
When the electronic tag is sensed by the RFID reader, a data set of the type $<$ EPC, Location, Time $>$ is generated, as shown in Table 1. Among them, EPC (Electronic Product Code) represents the electronic product code, which is unique. Location indicates the location of the object as it is scanned by the reader. Time indicates the time of the tuple, which is the scan time of the reader.

Table 1. Original RFID path data set

\begin{tabular}{|c|c|}
\hline EPC & RFID path data \\
\hline Epc1 & $\left(\mathrm{epc} 1,1_{1}, \mathrm{t}_{1}\right)\left(\mathrm{epc} 1,1_{1}, \mathrm{t}_{2}\right) \ldots \ldots\left(\mathrm{epc} 1, \mathrm{l}_{1}, \mathrm{t}_{10}\right)$ \\
\hline Epc2 & 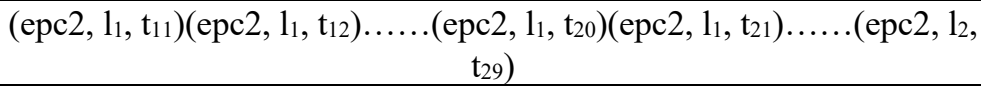 \\
\hline Epc3 & $\begin{array}{c}\left(\mathrm{epc} 3,1_{1}, \mathrm{t}_{12}\right) \ldots\left(\operatorname{epc} 3,1_{1}, \mathrm{t}_{18}\right)\left(\operatorname{epc} 3,1_{2}, \mathrm{t}_{19}\right) \ldots\left(\mathrm{epc} 3,1_{2}, \mathrm{t}_{22}\right)\left(\mathrm{epc} 3,1_{3},\right. \\
\left.\mathrm{t}_{23}\right) \ldots\left(\operatorname{epc} 3,1_{3}, \mathrm{t}_{30}\right)\end{array}$ \\
\hline & \\
\hline Epc500 & $\left(\mathrm{epc} 500,1_{1}, \mathrm{t}_{308}\right)\left(\mathrm{epc} 500,1_{1}, \mathrm{t}_{400}\right)\left(\mathrm{epc} 500,1_{3}, \mathrm{t}_{401}\right) \ldots\left(\mathrm{epc} 500,1_{3}, \mathrm{t}_{430}\right)$ \\
\hline
\end{tabular}

After observing the data in Table 1, it is seen that the RFID path is time dependent. Each location is accompanied by a time message. There have been more in-depth studies on common data sets. However, there is a certain difference between the RFID path data and the ordinary data, which is suitable for the research method of the common path. In general, it is not fully applicable to RFID path data.

The following are the characteristics of the RFID path data:

Massive: In the commercial sector, the flow of goods is frequent. The information of the object is collected by the reader, and the amount of data generated is very large.

Redundancy: Redundancy is more evident in RFID data. The reason for this is that the RF signal is constantly being sent by the reader. However, the coverage of the RF signal may overlap, and both spatial and temporal redundancy may occur.

Continuity: In the RFID application environment, a large number of tags often send information to the reader constantly, which enables the continuously transmitted data to be transmitted to the server in a certain time sequence, thereby forming a data stream with continuous nature. In some cases, data needs to be processed in a timely manner.

Dispersion: RFID is used in logistics management because the circulation of goods is very wide. For example, clothes produced in China can be sold to Europe, so RFID data is scattered in different places.

Time and space characteristics: In the process of collecting data, the electronic tag of the collected object changes its position at any time, so that the collected data includes both geographical factors and chronological order, that is, the spatiotemporal characteristics of the RFID data. This spatio-temporal information becomes another potential problem for RFID data mining. 


\subsection{Preprocessing of RFID data}

RFID data is exploding after the widespread adoption of radio frequency identification technology. How to store these massive data and save storage space, while meeting various query requirements, has prompted RFID data storage and management issues to become a research hotspot. In the practical application of car networking RFID, a large amount of path information is generated at any time. For example, a reader is installed at the intersection of the main street, and relevant RFID path data is generated at regular intervals. If the data is not preprocessed, the amount of data used for data mining and data compression is very large. The path data is processed from both geographic location and time information to improve the efficiency and accuracy of data compression.

The reader installed at the intersection will continuously scan the electronic tags in the range. If a car stays in a certain place for a longer period, the RFID system will generate more data tuples in the form of EPC, Location, and Time. The geographic location between these tuples is the same. These duplicate data are called redundant data. These redundant data need to be processed, and the original data shown in Table 1 can be converted into a static record of a shape (such as EPC, Location, Time_In, Timeout).

\subsection{RFID path big data compression in car networking}

According to reversibility, data compression is divided into two types: lossy compression and lossless compression. Based on the typical lossless compression algorithm LZW, it is improved and verified. Lossless compression refers to the removal of redundant information in the source, but it does not affect the information entropy. When decompressed, the compressed data can be fully recovered. The LZW compression algorithm was jointly proposed by Lemple-Ziv-Welch, which was formed on the basis of the classical compression algorithm LZ78. It is a practical algorithm. Figure 3 is a flow chart of the LZW algorithm.

LZW is a dictionary model compression algorithm. The initialization of the dictionary string table is to take advantage of all possible characters. During compression, if the current string has appeared in the dictionary table, the next character is read and added to the current string. Next, it is determined whether the matching character has appeared in the dictionary, and continues according to this method. Conversely, if the current string does not appear, the current string is added to the dictionary and the dictionary index of the largest matching string is output. In the same way, when the file is decompressed, as long as the dictionary string table synchronized with the compression process is maintained, the dictionary is restored, thereby realizing the decompression of the file.

After pre-processing the RFID path data, the path location time information is saved by data compression to reduce the storage space. The compression of the path information will follow the principle of LZW algorithm compression. The encoding of the terms in the dictionary is used to replace the string in the data to be compressed. 


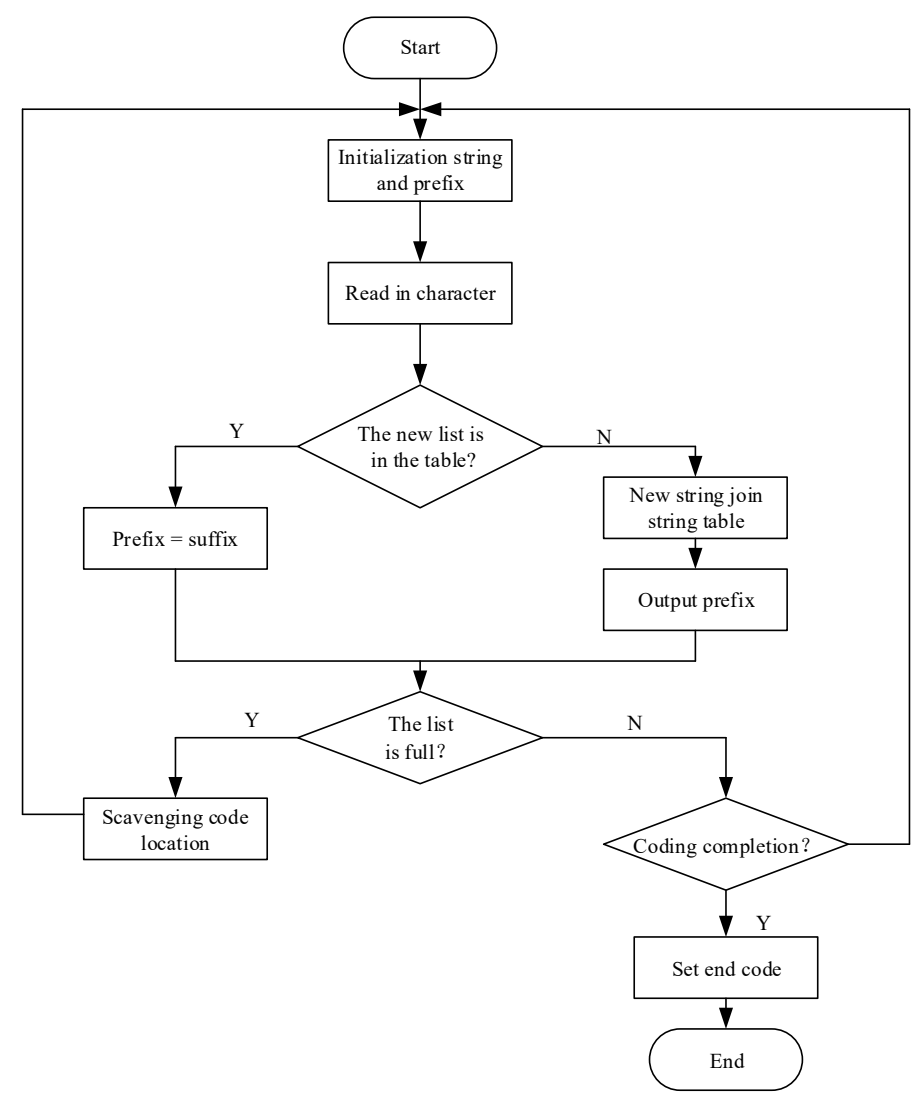

Fig. 3. Flow chart of LZW algorithm

Therefore, the longer the term in the dictionary, the higher the compression ratio will be. Therefore, increasing the storage capacity of the dictionary can effectively increase the compression ratio. However, the size of the dictionary is affected by the memory of the computer. In order to realize the storage function of RFID path data, a compression algorithm based on LZW is proposed, which is further improved to make it suitable for compression of RFID tag data.

The improved process of the LZW encoding algorithm is as follows:

Step1: The dictionary is initialized to a single character that may appear, and the current prefix $\mathrm{P}$ is initialized to be empty, so that the threshold is 1;

Step2: The current character $\mathrm{C}:=$ the next character in the character stream;

Step3: Is $\mathrm{P}+\mathrm{C}$ in the dictionary?

If "yes", extend $\mathrm{P}$ with $\mathrm{C}$, that is, let $\mathrm{P}:=\mathrm{P}+\mathrm{C}$;

If "No", the code word W corresponding to the current prefix $\mathrm{P}$ is output; $\mathrm{P}+\mathrm{C}$ is added to the dictionary; let $\mathrm{P}:=\mathrm{C}$;

Step4: Is the dictionary filled? 
If "yes", the compression ratio is calculated. If the compression ratio is greater than the threshold, the threshold = compression ratio is first used to continue compression; if the compression ratio is less than the threshold, the dictionary excess entries are deleted;

If "No", return to step 2.

The traditional LZW algorithm empties and rebuilds the dictionary when it is full. This results in a low compression efficiency at the beginning of each reconstruction. The threshold determination method will be improved to improve the dictionary compression ratio. When the dictionary is filled, a length of bit stream is tested. The test data stream is compressed using an existing dictionary, and the compression ratio of the data stream is obtained after compression. The formula of the compression ratio is expressed as:

Data compression ratio $=$ Compressed data stream bits $/$ Data bits before compression

By comparing with the specified threshold, when the compression ratio is greater than the threshold, the dictionary is used to compress and judge the remaining characters. When the resulting compression ratio is less than the threshold, the extra entries in the dictionary are deleted. According to a specific application environment, the improvement of the threshold judgment operation method is that the initial threshold is set to a fixed value of 1 , and then the threshold is judged. If the compression rate of the data stream is less than the threshold, the extra entries in the dictionary are deleted. If it is greater than the threshold, the new threshold is set to the compression ratio obtained by the test, and then the operation of the threshold judgment is continued. A threshold that is too large will remove the entry. If the operation of updating the dictionary is too frequent, the compression efficiency is lowered. If the threshold is too small, the dictionary will not be updated in time, which will still reduce the efficiency of compression. The improved threshold determination method will be more flexible. The dictionary is updated as needed, which increases the compression ratio and also reduces the time.

\section{Result Analysis and Discussion}

\subsection{Experimental environment and experimental data set}

Experimental hardware environment: Intel (R) Core (TM) i32. 4GHz CPU, 4GB of memory. Experimental software environment: Microsoft Windows 7. The experimental algorithm is implemented in Microsoft Visual $\mathrm{C}++6.0$ programming software.

The experimental data is the analog data generated by the RFID data generator. There are three main parts: the EPC unique identification code, time information and geographic location. The experimental data format is shown in Table 2. 
Table 2. Format of experimental data

\begin{tabular}{|c|c|c|}
\hline EPC code & Time information & $\begin{array}{c}\text { Number of geographical } \\
\text { locations }\end{array}$ \\
\hline Epc1 & $2016-10-0517: 59$ & Lbbb \\
\hline Epc2 & $2016-10-0518: 05$ & L07e \\
\hline Epc3 & $2016-10-0518: 11$ & L042 \\
\hline Epc4 & $2016-10-0518: 18$ & Lbbb \\
\hline Epc5 & $2016-10-0518: 26$ & L041 \\
\hline Epc6 & $2016-10-0518: 30$ & L061 \\
\hline Epc7 & $2016-10-0518: 37$ & L277 \\
\hline Epc8 & $2016-10-0518: 38$ & L1b9 \\
\hline Epc9 & $2016-10-0518: 48$ & L00c \\
\hline Epc10 & $2016-10-0518: 54$ & L041 \\
\hline Epc11 & $2016-10-0519: 01$ & L277 \\
\hline Epc12 & $2016-10-0519: 10$ & \\
\hline
\end{tabular}

\subsection{Verification of MBPrime encoding method}

When the amount of data is increased from 10,000 to 50,000, the storage method based on prime number coding (method 1) and the MBPrime code storage method (method 2) (without considering non-path attributes) in the traditional data management method are compared from two aspects respectively. The big data compression effect and the corresponding time of the data query are shown in Figure 4.

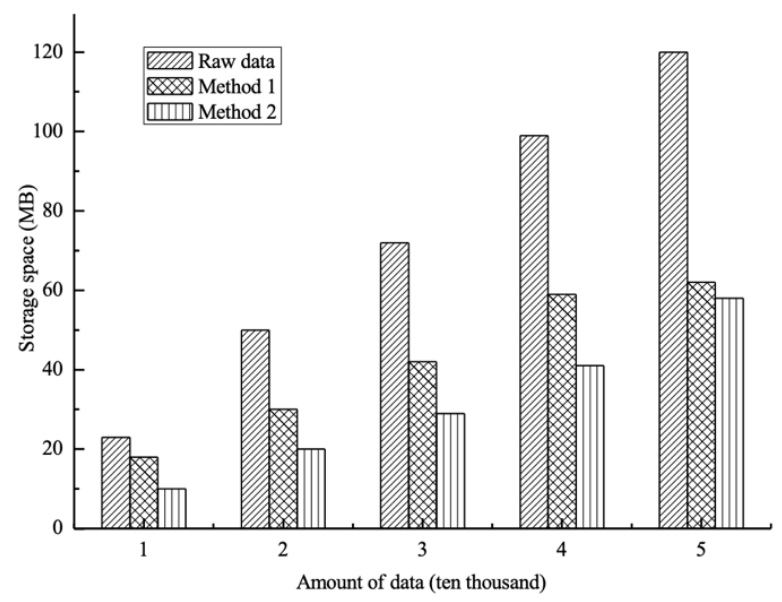

Fig. 4. Block diagram of the hardware of the control system

The compression ratio of the prime coding method is significantly improved compared with the original data storage. The prime coded storage mode also has a good compression effect. However, it is slightly insufficient compared to the MBPrime coded storage method. The number of primes required by the MBPrime encoding method is always less than or equal to the number of primes used in the 
PrimeLabel encoding method. It can be seen that the RFID location information will have a good effect when encoded by the MBPrime method.

The following is a comparison of query performance in three storage scenarios. The experiment performs object tracking query on RFID path data, which is a typical object tracking query. The motion path of the specified object of the EPC code is found, as shown in Figure 5.



Fig. 5. Response time of object tracking query

As can be seen from Figure 5, it takes a long time to track the object on the original data set. In particular, more and more time is required as the amount of data increases. The prime coded storage model is compared to the MBPrime coded storage model. When the amount of data is small, the query performance is small. As the amount of data increases, the time of prime coding increases faster than the time of the MBPrime coded storage model.

\subsection{Verification of R-LZW compression algorithm}

The experimental data is 100,000 preprocessed path data sets to verify the efficiency of the improved algorithm. Several commonly used compression algorithms are used to compress it. Then, it is compared with the performance of the R-LZW compression algorithm. The compression ratio and the time consumed by compression are the main comparison criteria. Table 3 compares the performance of each algorithm.

Table 3. Comparison of performance of each algorithm

\begin{tabular}{|c|c|c|}
\hline Algorithm & Compression ratio (\%) & Time consumption (S) \\
\hline LZ77 & 39 & 4.2 \\
\hline LZ78 & 49 & 4 \\
\hline LZW & 59 & 3 \\
\hline R-LZW & 66 & 2.5 \\
\hline
\end{tabular}


From the experimental results in Table 3, the improved R-LZW algorithm has a higher compression ratio than the LZ77, LZ78, and LZW compression algorithms. Compression takes less time than several other common compression algorithms. From the original LZ77 algorithm to the final improved R-LZW algorithm, the compression ratio increased by $28 \%$. Therefore, it is found from this experiment that the improved algorithm is more suitable for compressing RFID path data. It not only increases the space storage rate of RFID tags, but also shortens the compression time.

The second experiment will verify the threshold judgment method in the R-LZW algorithm. Four data sets are used, namely $1 \times 104,2 \times 104,3 \times 104,4 \times 104$. The data set was experimentally verified using two different threshold judgment methods. The results are shown in Figure 6.

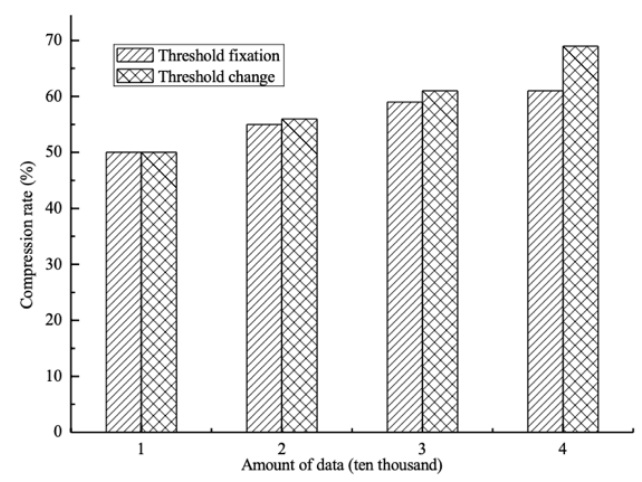

Fig. 6. Comparison of different threshold judgment methods

When the amount of data is relatively small, there is no significant change in the compression ratio between the threshold and the threshold change. However, when the amount of data is gradually increased, the compression rate in the case where the threshold is changed is slightly higher than the fixed threshold. The experimental results demonstrate the feasibility of this method for RFID path data processing and the efficiency of compression.

\section{Conclusion}

The big data compression technology based on car networking is studied. First, the concepts of RFID technology and car networking were introduced. Then, the generation and recognition process of RFID path data, as well as the spatio-temporal characteristics of the data are described, and the path data is preprocessed. When the location information of the RFID path is compressed, the path data is encoded by the MBPrime coding method. The R-LZW algorithm is used in compressing all path information data, and the threshold value judgment method is improved. Finally, the algorithm is validated using simulated data. The results show that the MBPrime coding method can efficiently encode the location information in the RFID path data. R-LZW can compress and store all the information of the path. 


\section{References}

[1] Kim, D. H., Choi, K. H., Li, K. J., Lee, Y. S. (2015). Performance of vehicle speed estimation using wireless sensor networks: a region-based approach. Journal of Supercomputing, 71(6): 2101-2120 https://doi.org/10.1007/s11227-014-1306-7

[2] Grishentsev, A. Y., Korobeynikov, A. G., Korikov, C. C., Velichko, E. N. (2016). Method for compression of optical observation data based on analysis of differential structure. Optical Memory \& Neural Networks, 25(1): 32-39 https://doi.org/10.310 $\underline{3 / \mathrm{S} 1060992 X 16010045}$

[3] Heo, G., \& Jeon, J. (2017). A study on the data compression technology-based intelligent data acquisition (idaq) system for structural health monitoring of civil structures. Sensors, 17(7): 1620 https://doi.org/10.3390/s17071620

[4] He, W., Yan, G., Da Xu, L. (2014). Developing vehicular data cloud services in the IoT environment. IEEE Transactions on Industrial Informatics, 10(2): 1587-1595 https://doi.org/10.1109/TII.2014.2299233

[5] Amadeo, M., Campolo, C., Molinaro, A. (2016). Information-centric networking for connected vehicles: a survey and future perspectives. IEEE Communications Magazine, 54(2): 98-104 https://doi.org/10.1109/MCOM.2016.7402268

[6] Chen, M., Zhang, Y., Hu, L., Taleb, T., Sheng, Z. (2015). Cloud-based wireless network: Virtualized, reconfigurable, smart wireless network to enable $5 \mathrm{G}$ technologies. Mobile Networks and Applications, 20(6): 704-712 https://doi.org/10.1007/s11036-015-0590-7

[7] Checko, A., Christiansen, H. L., Yan, Y., Scolari, L., Kardaras, G., Berger, M. S., \& Dittmann, L. (2015). Cloud RAN for mobile networks - A technology overview. IEEE Communications surveys \& tutorials, 17(1): 405-426 https://doi.org/10.1109/CO MST.2014.2355255

[8] Da, Xu, L., He, W., Li, S. (2014). Internet of things in industries: A survey. IEEE Transactions on industrial informatics, 10(4): 2233-2243 https://doi.org/10.1109/ $\underline{\text { TII.2014.2300753 }}$

\section{Author}

Guohua Xiong is a Researcher of Guandong Construction Polytechnic, Guangdong, China. His research interests include Car Networking.

Article submitted 26 October 2018. Resubmitted 28 November 2018. Final acceptance 12 December 2018. Final version published as submitted by the authors. 